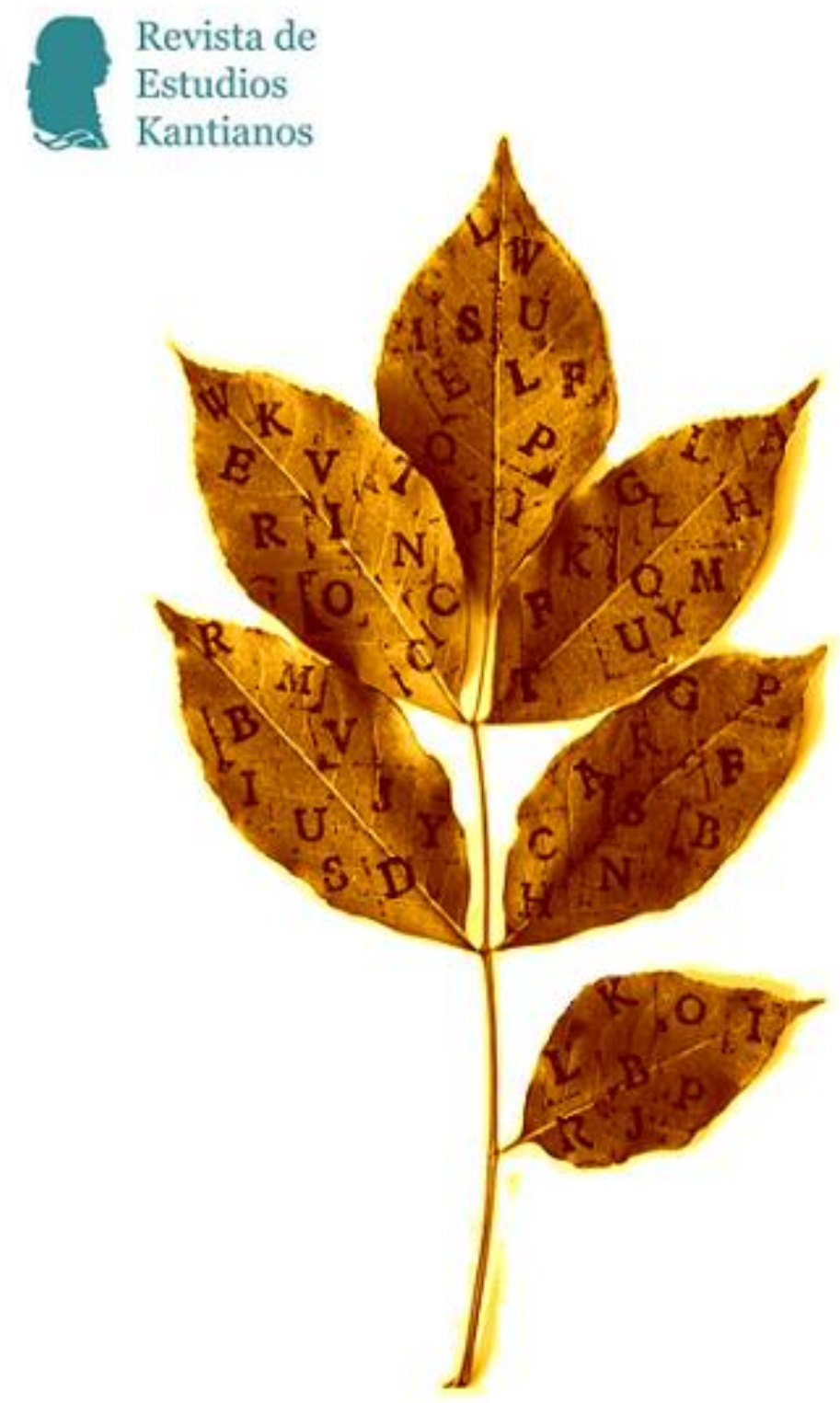


Revista de Estudios Kantianos 


\section{Revista de Estudios Kantianos}

Publicación internacional de la Sociedad de Estudios Kantianos en Lengua Española Internationale Zeitschrift der Gesellschaft für Kant-Studien in Spanischer Sprache International Journal of the Society of Kantian Studies in the Spanish Language

\section{Dirección}

Pedro Jesús Teruel, Universitat de València

pedro.teruel@uv.es

Hernán Pringe, CONICET-Universidad de Buenos Aires/

Universidad Diego Portales, Santiago de Chile

hpringe@gmail.com

\section{Secretario de edición}

Fernando Moledo, Universidad de Buenos Aires - CONICET

fernandomoledo@filo.uba.ar

\section{Secretaria de calidad}

Marcela García, Universidad de Morelia, México

garciar.marcela@gmail.com

\section{Editores científicos}

Jacinto Rivera de Rosales, UNED, Madrid

Claudia Jáuregui, Universidad de Buenos Aires

Vicente Durán, Pontificia Universidad Javeriana, Bogotá

Julio del Valle, Pontificia Universidad Católica del Perú, Lima

Jesús Conill, Universitat de València

Gustavo Leyva, Universidad Autónoma de México, México D. F.

María Xesús Vázquez Lobeiras, Universidade de Santiago de Compostela

Wilson Herrera, Universidad del Rosario, Bogotá

Pablo Oyarzun, Universidad de Chile, Santiago de Chile

Paula Órdenes Azúa, Universität Heidelberg 


\section{Comité científico}

Juan Arana, Universidad de Sevilla

Reinhardt Brandt, Philipps-Universität Marburg

Mario Caimi, Universidad de Buenos Aires

Monique Castillo, Université de Paris-Est

Adela Cortina, Universitat de València

Bernd Dörflinger, Universität Trier

Norbert Fischer, Universität Eichstätt-Ingolstadt

Miguel Giusti, Pontificia Universidad Católica del Perú

Dulce María Granja, Universidad Nacional Autónoma de México

Christian Hamm, Universidad Federal de Santa María, Brasil

Dietmar Heidemann, Université du Luxembourg

Otfried Höffe, Universität Tübingen

Claudio La Rocca, Università degli Studi di Genova

Juan Manuel Navarro Cordón, Universidad Complutense, Madrid

Carlos Pereda, Universidad Nacional Autónoma de México

Gustavo Pereira, Universidad de la República, Uruguay

Ubirajara Rancan de Azevedo, Universidade Estadual Paulista, Brasil

Margit Ruffing, Johannes Gutenberg-Universität Mainz

Gustavo Sarmiento, Universidad Simón Bolívar, Venezuela

Sergio Sevilla, Universitat de València

Roberto Torretti, Universidad Diego Portales, Santiago de Chile

Violetta Waibel, Universität Wien

Howard Williams, University of Aberystwyth

Allen W. Wood, Indiana University

Diseño, revisión de estilo y maqueta

Josefa Ros Velasco, Harvard University, Cambridge (MA)

\section{Corrector}

Aldo Perán, Universidad Diego Portales, Santiago de Chile

\section{Entidades colaboradoras}

Sociedad de Estudios Kantianos en Lengua Española (SEKLE)

Departament de Filosofia de la Universitat de València

Instituto de Humanidades, Universidad Diego Portales

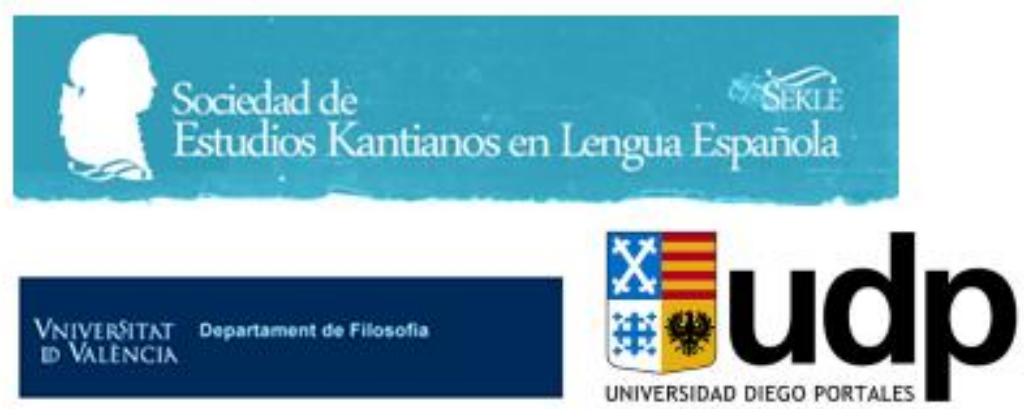




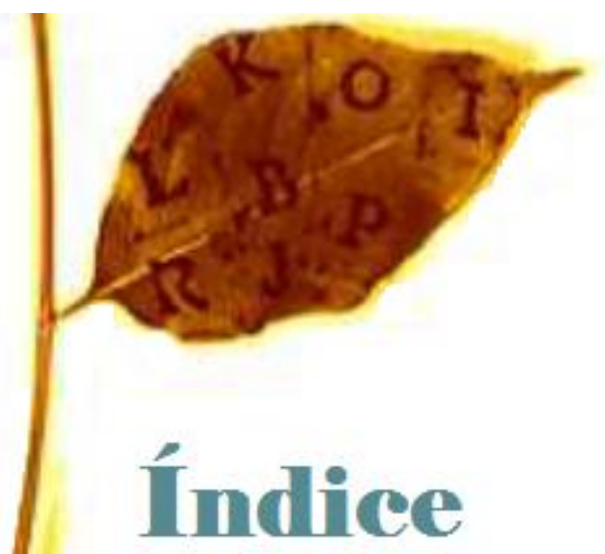

\section{Artículos}

125 Los deberes del amor en la doctrina kantiana de la virtud Bernd Dörflinger DOI 10.7203/REK.2.2.10807

135 Towards a new conception of metaphysics: Lambert's criticism on Wolff's mathematical method

Gesa Wellmann

DOI 10.7203/REK.2.2.10724

149 Acerca del alcance objetivo de las prescripciones metodológicas de la función regulativa de la razón teórica en la Crítica de la razón pura de Kant

Martín Arias Albisu

DOI 10.7203/REK.2.2.8432

168 Formas de autoconciencia en la "Reflexión de Leningrado" Matías Hernán Oroño

DOI 10.7203/REK.2.2.8799

179 Kants Konzeption kosmologischer Freiheit - ein metaphysischer Rest?

Christian Krijnen

DOI 10.7203/REK.2.2.10407

\section{Informe}

196 VII Coloquio Multilateral-Kant 2017

Paula Órdenes Azúa

DOI 10.7203/REK.2.2.10756 


\section{Reseñas}

200 Alfredo Ferrarin: Il pensare e l'io. Hegel e la critica di Kant. Roma, Carocci Editore, 2016, 244 pp. ISBN: 978-88-430-8246-9.

Luigi Filieri

DOI 10.7203/REK.2.2.10764

205 Mario Caimi, Ileana Beade, José González Ríos, Macarena Marey, Fernando Moledo, Mariela Paolucci, Hernán Pringe y Marcos Thisted: Diccionario de la filosofía crítica kantiana. Buenos Aires, Colihue, 2017, 507 pp. ISBN: 978-950-563-450-7.

Miguel Herszenbaun

DOI 10.7203/REK.2.2.10499

210 Paula Órdenes Azúa y Daniela Alegría: Kant y el Criticismo: pasado, presente, y ¿futuro?, Porto Alegre, Editora Fi, 2015, 303 pp. ISBN: 978-85-66923-52-0.

Constanza Terra

DOI 10.7203/REK.2.2.10777

212 Juan Ormeño Karzulovic y Miguel Vatter (eds.): Forzados a ser libres. Kant y la teoría republicana del derecho. Santiago, Fondo de Cultura Económica, 2017, 243 pp. ISBN: 978956-289-149-3.

Cristóbal Olivares

DOI 10.7203/REK.2.2.10762

215 Immanuel Kant (ed. y trad. Gonzalo Serrano): La Deducción trascendental y sus inéditos, 1772-1788. Bogotá, Universidad Nacional de Colombia, 2014, 321 pp. ISBN: 978-95-876195-39.

Pedro Stepanenko

DOI 10.7203/REK.2.2.10763

\section{Semblanza}

220 Juan Adolfo Bonaccini (16 de mayo de 1965 / 10 de julio de 2016)

Daniel Omar Perez

DOI 10.7203/REK.2.2.10783

\section{Convocatorias y normas para autores}

223 Kant en México

DOI 10.7203/REK.2.2.10863

224 IV Congreso Internacional de la SEKLE, Valencia, 2018

DOI 10.7203/REK.2.2.10863

227 VIII Coloquio Multilateral-Kant 2018

DOI 10.7203/REK.2.2.10863

229 Normas para autores

DOI 10.7203/REK.2.2.10863 


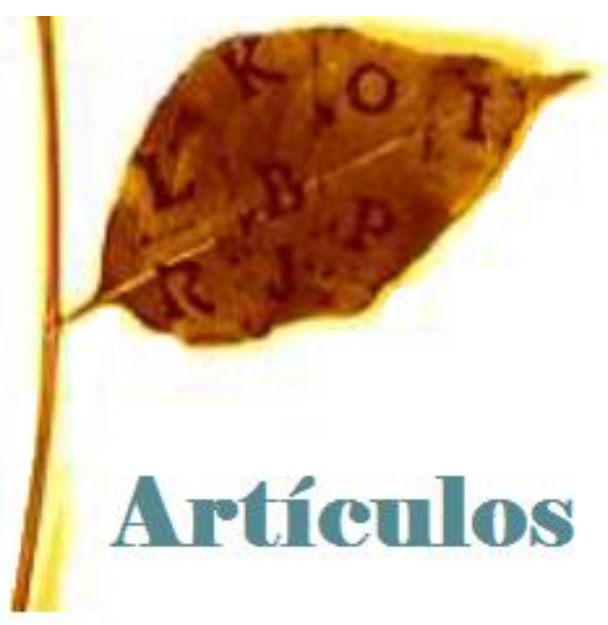




\title{
Acerca del alcance objetivo de las prescripciones metodológicas de la función regulativa de la razón teórica en la Crítica de la razón pura de Kant
}

\author{
MARTÍN ARIAS ALBISU ${ }^{1}$
}

\begin{abstract}
Resumen
En el "Apéndice a la dialéctica trascendental" de su Crítica de la razón pura, Kant presenta prescripciones metodológicas para el conocimiento empírico. Expondré los dos conjuntos de prescripciones más importantes de esa metodología: a) la necesidad de introducir conceptos "teóricos"; b) el uso hipotético de la razón y la exigencia de sistematicidad, que se refieren a leyes empíricas y a los conceptos vinculados con tales leyes. Cada uno de estos conjuntos de prescripciones tiene una dimensión objetiva propia, esto es, su aplicación es condición de posibilidad de la formación y validez objetiva de determinadas clases de leyes y/o conceptos. Mi objetivo es presentar las diferencias y relaciones mutuas entre esas diferentes dimensiones objetivas.
\end{abstract}

Palabras clave: Crítica de la razón pura, ciencia empírica, regulativo, metodología, objetividad

\section{On the Objective Scope of the Methodological Prescriptions of the Regulative Function of Theoretical Reason in Kant's Critique of Pure Reason}

\begin{abstract}
In the "Appendix to the Transcendental Dialectic" of his Critique of Pure Reason, Kant presents methodological prescriptions for empirical knowledge. I will expound the two most important sets of prescriptions of this methodology: a) the necessity of introducing "theoretical" concepts; b) the hypothetical use of reason and the demand for systematicity, which refer to empirical laws and to the concepts related to such laws. Each of these sets of prescriptions has its own objective dimension, that is, its application is a condition of the possibility of the formation and objective validity of determinate kinds of laws and/or concepts. My aim is to present the differences and mutual relations between these different objective dimensions.

Keywords: Critique of Pure Reason, empirical science, regulative, methodology, objectivity
\end{abstract}

Introducción

En el extenso cuerpo de la "Dialéctica trascendental" de su Crítica de la razón pura (KrV, 1781/1787), Kant intentó justificar la tesis según la cual las ideas de la razón teórica no pueden brindar conocimiento de los objetos suprasensibles alma, mundo y Dios. Al final de esa división de la Crítica, Kant introdujo una concisa sección denominada "Apéndice a la dialéctica trascendental" ("Anhang zur transscendentalen Dialektik" = AtD). Esta sección contiene dos partes, a saber, "Del uso regulativo de las ideas de la razón pura" (AtD1) y "Del propósito último de la dialéctica natural

${ }^{1}$ Universidad Nacional del Rosario - CONICET 
de la razón humana" (AtD2). En ellas se expone el legítimo uso regulativo de las ideas de la razón teórica con respeto al conocimiento empírico del entendimiento. ${ }^{2}$

Varios comentaristas señalaron que en el texto del AtD hay una contradicción o, al menos, cierta tensión, entre una concepción metodológica o subjetiva de la función regulativa de la razón teórica y otra transcendental u objetiva. ${ }^{3}$ A diferencia de tales comentaristas, estableceré una clara demarcación entre las concepciones subjetiva y objetiva de la función regulativa de la razón teórica que permitirá mostrar que ellas no son contradictorias. La novedad de mi contribución consiste en que, a fin de determinar la diferencia y la articulación entre las dos mencionadas concepciones, tendré en cuenta las peculiaridades de las prescripciones metodológicas exigidas por la función regulativa de la razón teórica que considero más importantes, a saber: i) la necesidad de introducir ciertas ideas de la razón que denomino 'conceptos teóricos' (como el de 'flogisto', que se utilizaba en la química flogística de la época de Kant); ii) el uso hipotético de la razón con sus cinco criterios para la admisión y/o evaluación de las hipótesis de leyes empíricas que emplean conceptos teóricos y empíricos (aquí examinaré, fundamentalmente, las que emplean al menos un concepto teórico); y iii) la exigencia de máxima unidad y extensión sistemáticas para esa clase de hipótesis y esas clases de conceptos.

Las prescripciones ii) y iii) están íntimamente vinculadas. Efectivamente, la exigencia de sistematicidad planteada por la prescripción iii), en la medida en que se ejerce sobre hipótesis de leyes empíricas, puede considerarse como un criterio para la evaluación de estas hipótesis, el cual se agrega a los cinco criterios de esa clase que, de acuerdo con la prescripción ii), deben aplicarse necesariamente. De hecho, como se verá, la posibilidad de integrar una hipótesis de ley empírica en un sistema de esas leyes es uno de los criterios que se deben emplear para evaluar el valor de verdad de esa hipótesis. ${ }^{4}$ Por tal motivo, las prescripciones ii) y iii) serán analizadas aquí conjuntamente.

El estudio de esta problemática me permitirá sostener la tesis según la cual las prescripciones metodológicas i) y ii)-iii) determinan concepciones objetivas de la función regulativa de la razón teórica como correlatos de cada una de ellas que son, al menos parcialmente, diferentes, aunque existen conexiones y articulaciones entre las mismas. ${ }^{5}$ Por 'correlato objetivo' o 'dimensión objetiva' de una prescripción metodológica entiendo aquí la(s) clase(s) determinada(s) de leyes y/o conceptos cuya formación y validez objetiva tienen como condición de su posibilidad la aplicación de esa prescripción. A su vez, como intentaré mostrar, tales leyes y conceptos son condiciones de posibilidad de ciertos tipos de experiencia.

A continuación examinaré las prescripciones i) y ii)-iii) en ese orden y en dos secciones diferentes.

\footnotetext{
${ }^{2}$ A fin de simplificar, me referiré al uso regulativo de las ideas de la razón teórica con la expresión 'función regulativa de la razón teórica'.

${ }^{3}$ Acerca de la tensión o contradicción entre una concepción subjetiva y otra objetiva de la función regulativa de la razón teórica, cf. Kemp Smith 1923/2003: 543-561; Bennett 1974: 275 ss.; Kitcher 1986: 207. Por otro lado, los textos de Kant se citan a partir de la Akademie-Ausgabe y según las siglas establecidas en el sitio web de la Kant-Forschungsstelle de la Universidad de Mainz (http://www.kant.uni-mainz.de/ks/abhandlungen.html). La primera Crítica $(\mathrm{KrV})$ es citada, como es habitual, de acuerdo con la paginación de la primera $(\mathrm{A}=1781)$ y segunda $(\mathrm{B}=1787)$ ediciones. Véase la bibliografía para más detalles y una indicación de las traducciones castellanas utilizadas. Modifiqué algunos pasajes de esas traducciones para ofrecer versiones más fieles al original alemán.

${ }^{4}$ Cf. infra, n. 9 y Sección 2. Por otro lado, una argumentación presentada en un artículo de Mario Caimi permite mostrar la complementariedad de las dimensiones subjetiva y objetiva de la exigencia regulativa de sistematicidad referida fundamentalmente a los conceptos empíricos (cf. Caimi 1995: 311-317 y Sección 2).

${ }_{5}^{5}$ Acerca de estas prescripciones y las relaciones entre ellas, véase Arias Albisu 2015. Por otro lado, el principio trascendental de la facultad de juzgar reflexionante de la Crítica de la facultad de juzgar (KU) no puede examinarse en este artículo por razones de espacio.
} 
1. La exigencia regulativa de introducción de conceptos teóricos y su dimensión objetiva

La exigencia regulativa de introducir las ideas de la razón que aquí denomino 'conceptos teóricos' es presentada por Kant en el quinto párrafo del AtD1 (A645-646/B673-674). ${ }^{6}$ Efectivamente, en ese párrafo Kant presenta ideas de la razón como las de tierra pura y de sales. Esas ideas no tienen, en sentido estricto, objetos que les correspondan adecuadamente en la naturaleza, dado que se originan en la razón teórica. Sin embargo, es necesario introducir ideas como las mencionadas para "determinar debidamente la participación que cada una de esas causas naturales [MA: tierra pura, etc.] tiene en el fenómeno". De esta manera, "todas las materias se reducen a tierras (por así decir, el mero peso), a sales y cuerpos combustibles (que vienen a ser la fuerza), y finalmente a agua y aire, como vehículos (por así decir, como máquinas por medio de las cuales las precedentes actúan)". Se procuran explicar así, mecánicamente, los efectos químicos recíprocos de esas materias.

Los ejemplos de conceptos teóricos ofrecidos por Kant pertenecen a la química flogística de la época. De hecho, la expresión 'cuerpos combustibles' [brennliche Wesen] hace referencia al principio de la inflamabilidad o "flogisto". A fin de explicar las concisas afirmaciones de Kant, expondré primeramente, en general, la noción de concepto de teórico, y mostraré luego más detalladamente la vinculación entre estos conceptos teóricos y la química.

Para Kant, los conceptos teóricos se originan, en lo que a "la completa pureza" se refiere, en la razón teórica ( $\mathrm{KrV}$, A646/B674). El entendimiento emplea sus categorías y conceptos empíricos para hacer posible, por ejemplo, una experiencia de la calcinación de un trozo de plomo. Sin embargo, a fin de explicar este proceso es necesario que la razón realice una determinada labor conceptual. Semejante labor consiste en interpretar procesos como el mencionado como el resultado de la operación de ciertos elementos puros contenidos en objetos empíricos. De esta manera, se intenta mostrar que las propiedades y procesos químicos de esos objetos dependen de la presencia y la operación de esos elementos. Tales elementos son representados por la razón de manera pura, esto es, sin tener en cuenta los otros elementos con los cuales ellos se encuentran siempre mezclados en la naturaleza. Por el contrario, el entendimiento sólo puede representar objetos empíricos en los cuales tales elementos están mezclados unos con otros. Denominaré a los conceptos que son representaciones de estos elementos puros, como adelanté, 'conceptos teóricos', y a esos elementos puros mismos, 'objetos teóricos'?

Los objetos teóricos son postulados en hipótesis de leyes empíricas científicas que incluyen en su formulación los conceptos teóricos que se refieren a los primeros. Tales objetos teóricos no son ni objetos empíricos ni construcciones de conceptos matemáticos en la intuición pura. Más bien, los objetos teóricos son entidades puramente ideales. Ellos no están sometidos a todas las variaciones que sufren los objetos empíricos en virtud de estar compuestos por mezclas de diferentes elementos puros. De hecho, los objetos teóricos sólo pueden variar de determinadas maneras, las cuales son previstas en la formulación de la hipótesis que se refiere a ellos. Además, como los objetos teóricos no son postulados para explicar un fenómeno singular, sino una(s) clase(s) determinada(s) de fenómenos, estos objetos hacen posible una explicación no sólo precisa, sino también económica, de esa(s) clase(s) de fenómenos. Aunque los fenómenos que, según la hipótesis, contienen como elemento puro

${ }^{6}$ Anteriormente, esto es, al comienzo del AtD1, Kant expone de manera preliminar una concepción de las ideas de la razón teórica relacionada con la exigencia de sistematicidad del conocimiento empírico (cf. KrV, A642-645/B670-673). Esta concepción se aplica fundamentalmente a dos diferentes clases de estas ideas, a saber, las ideas como principios (expuestas más adelante en AtD1) y como entes de razón (presentadas en AtD2). La exigencia regulativa de sistematicidad será examinada en la Sección 2.

${ }^{7}$ La designación "concepto teórico" es empleada por Buchdahl (1965, 1969 y 1992), Wartenberg (1979 y 1992) y Brittan (1992) Buchdahl también emplea las designaciones "concepción teórica" (1965) y "constructo teórico" (1967). En cambio, Rauscher hace referencia a "ideas mundanas" (2010). Aquí sigo principalmente a Brittan, quien también emplea el concepto de "objeto teórico" en un sentido similar al que aquí le concedo. Por otro lado, Carrier (2001) y McNulty (2015) estudian el empleo de las ideas de la razón que denomino "conceptos teóricos" en el ámbito de la química de la época. 
el objeto teórico postulado por ella pueden ser diferentes, ese objeto teórico pensado como contenido en ellos es por definición siempre el mismo. Por tal motivo, un descubrimiento acerca de un objeto teórico es válido para todos los fenómenos en los que se piensa que ese objeto está contenido, así como para todos los fenómenos vinculados por la hipótesis en cuestión con los primeros. ${ }^{8}$

En su Crítica de la razón pura, Kant se ocupa del problema de la validez objetiva de ciertos tipos de conceptos - principalmente las categorías - que no son sino los conceptos empíricos y los matemáticos. Aquí es de interés presentar solamente una interpretación del sentido en el que los conceptos teóricos pueden adquirir cierta validez objetiva. ${ }^{9}$ Como estos conceptos se refieren a elementos puros que en la naturaleza se encuentran únicamente mezclados con otros, no es posible que a esos conceptos les correspondan intuiciones enteramente adecuadas. Sin embargo, justamente el hecho de que los elementos puros representados por conceptos teóricos sean entendidos, por definición, como contenidos en objetos empíricos, puede considerarse como un criterio de una cierta validez objetiva de tales conceptos. Pues, de esta manera, los conceptos teóricos pueden referirse efectivamente a intuiciones empíricas, aunque ellas no sean enteramente adecuadas como, por ejemplo, en el caso de los conceptos empíricos. Por otro lado, ya se señaló que los objetos teóricos son postulados en hipótesis de leyes empíricas científicas que emplean conceptos teóricos y procuran explicar los fenómenos dados. Por tanto, estimo que esta validez objetiva de los conceptos teóricos también depende de que las hipótesis que los emplean sean puestas a prueba exitosamente mediante experimentos. ${ }^{10}$

A continuación indicaré cuál es el tipo de ciencia que, según Kant, requiere el empleo de conceptos teóricos. Como adelanté en el pasaje mencionado al principio de esta Sección, en el que se introduce la noción de concepto teórico, la ciencia considerada es la química (A645-646/B673-674). Kant presenta en AtD1 otros ejemplos de ejercicio de la función regulativa de la razón teórica en el ámbito de esa disciplina. ${ }^{11}$ Friedman ha mostrado que durante el período crítico Kant adhería a la química flogística tradicional desarrollada especialmente por Georg E. Stahl (1659-1734). ${ }^{12}$ En un conocido pasaje del "Prólogo a la segunda edición" de la primera Crítica (BXII-XIV), Kant vincula los experimentos de, entre otros, Stahl, con una "revolución del modo de pensar" que puso a la ciencia de la naturaleza en la carretera de la genuina ciencia. ${ }^{13}$ Kant indica que tiene en cuenta la ciencia de la naturaleza únicamente en tanto que está basada en principios empíricos. Según el nuevo modo de pensar, en la ciencia de la naturaleza así entendida la razón debe dirigirse a la naturaleza con principios y experimentos diseñados de acuerdo a estos. De este modo, la razón "es instruida por ésta [MA: la naturaleza], pero no en calidad de un escolar que deja que el maestro le diga cuanto quiera, sino [en calidad] de un juez en ejercicio de su cargo, que obliga a los testigos a responder a las preguntas que él les plantea" (BXIII). La oposición entre la escucha atenta de lo que dice el maestro y el planteamiento de la obligación de responder a ciertas preguntas por parte del juez hace referencia, como está claro en el contexto, a la oposición entre 'observaciones contingentes' hechas sin ningún plan previo y la efectuación de experimentos de acuerdo con un cierto planeamiento. Puede

\footnotetext{
${ }^{8}$ Brittan expresa una tesis similar cuando afirma que "los objetos teóricos son como los matemáticos, enteramente representativos de la clase a la que pertenecen" (Brittan 1992: 176).

${ }^{9}$ Los conceptos tienen validez objetiva cuando, a grandes rasgos, tienen intuiciones empíricas que les corresponden. Cf. KrV, A238-246/B297-302

${ }^{10}$ Véanse al comienzo de esta Sección las tesis de Kant sobre la necesidad de determinar la participación de cada 'causa natural' (concepto u objeto teórico) en el fenómeno, y de explicar las interacciones químicas de las materias (en infra, n. 13 presento un experimento mencionado por Kant). En la Sección 2 mostraré que las hipótesis que emplean conceptos teóricos pueden tornarse más probables cuando son puestas a prueba empíricamente con éxito, pero nunca pueden alcanzar una necesidad estricta, sino sólo regulativa.

${ }^{11}$ Cf. KvR, A652-653/B680-681 y A657/B685. Cf. Sección 2.

${ }^{12}$ Friedman 1992: 240-242 y 264-267. Cf. infra, n. 17.

${ }^{13}$ También se mencionan experimentos de Galileo y Torricelli. Cf. infra, n. 17
} 
concluirse que la clase de disciplina que requiere conceptos teóricos es, al menos, la química experimental de Stahl. ${ }^{14}$

Un análisis de algunos pasajes del "Prólogo" a los Primeros principios metafísicos de la ciencia de la naturaleza (MAN, 1786), permitirá profundizar la comprensión del problema del tipo de ciencia que emplea conceptos teóricos. Allí se afirma que la "doctrina de la naturaleza" se divide en "ciencia de la naturaleza" y en "doctrina histórica de la naturaleza". Esta última contiene "hechos sistemáticamente ordenados de las cosas de la naturaleza" y se divide a su vez en "descripción de la naturaleza" e "historia de la naturaleza" (MAN, AA 04: 468). ${ }^{15}$

La ciencia de la naturaleza, que es la que aquí nos interesa, puede ser ciencia o bien de un modo propio o bien de uno impropio. La ciencia de la naturaleza en sentido propio del término, esto es, la física matemática, contiene una parte pura. Esta parte pura está compuesta por leyes de la naturaleza que son de carácter a priori y alcanzan entonces certeza apodíctica. Tal parte pura consta de una parte trascendental y una parte metafísica. La parte trascendental contiene las leyes a priori que determinan la forma de una naturaleza en general. Estas leyes son los principios del entendimiento puro expuestos en la primera Crítica (Krv, A148/B187 ss.). La parte metafísica es una metafísica de la naturaleza corpórea y consiste en el conocimiento a priori acerca de la totalidad de los objetos corpóreos que puede alcanzarse introduciendo un concepto empírico muy general de materia. Esta metafísica es presentada en MAN y fundamenta la aplicación adecuada de la matemática a los fenómenos del sentido externo (cf. MAN, AA 04: 468-473).

La ciencia de la naturaleza en sentido impropio, esto es, al menos, la química, contiene únicamente leyes basadas en la experiencia (MAN, AA 04: 468). La química es una ciencia en sentido impropio porque no posee una parte pura y la matemática no puede aplicarse adecuadamente dentro de su ámbito. ${ }^{16}$ Por otro lado, considero que la química es una ciencia, aunque en un sentido impropio, porque puede ser "arte sistemático o doctrina experimental" (MAN, AA 04: 471). En primer lugar, Kant conecta la sistematicidad con la cientificidad en la $\mathrm{KrV}$ (832/B860 ss.) y en MAN (AA, 04: 467). ${ }^{17}$ En segundo lugar, la conexión entre cientificidad y carácter experimental en, por ejemplo, la química de Stahl, se indica en el pasaje del "Prólogo a la segunda edición" de KrV (BXIIXIV) y que comenté anteriormente. Además, al comienzo de esta Sección se expuso una conexión entre experimentación y empleo de conceptos teóricos en el ámbito de esa química. Las leyes empíricas científicas que emplean conceptos teóricos pertenecientes a la química de Stahl, tal como el de flogisto, se deben poner a prueba mediante experimentos. Estimo, entonces, que los conceptos teóricos se emplean en la ciencia en sentido impropio del término, y que este empleo, junto con la aplicación de la exigencia regulativa de sistematicidad, explican el carácter científico de este tipo de

\footnotetext{
${ }^{14}$ En el mencionado pasaje del "Prólogo a la segunda edición" de su Crítica de la razón pura, Kant alude a un experimento realizado por Stahl. Kant afirma que el químico alemán "transformó metales en cal y ésta otra vez en metal, quitándoles algo y dándoselo de nuevo" (BXII-XIII). Stahl en primer lugar calcinó plomo para producir cal o ceniza de plomo (la cal o ceniza de un metal era concebida como un tipo de tierra, y hacía referencia a lo que hoy se denomina "óxido" de un metal). En este proceso, aparentemente, el flogisto contenido en el plomo fue removido. Posteriormente, Stahl quemó carbón en presencia de la cal de plomo y descubrió que esta última se transformó en un trozo metálico de plomo con sus características distintivas. Tal proceso se explicaría por la liberación del flogisto contenido en el carbón y su absorción por parte de la cal de plomo. Este experimento permitiría concluir que todas las calcinaciones y combustiones se explican por el mismo mecanismo de liberación del flogisto, y que el flogisto es el mismo en todos los reinos de la naturaleza, por ejemplo en el mineral (plomo) y en el vegetal (carbón). En otras palabras, este experimento sustentaría la hipótesis siguiente: el flogisto es el principio de la inflamabilidad. Es claro que, según Kant, lo que Stahl "quitó" y "dio de nuevo" es el objeto teórico "flogisto". Sigo las exposiciones de este experimento ofrecidas por Carrier y McNulty (cf. Carrier (2001: 217-218) y McNulty (2015: 5-6). Cf. también Stahl (1718: 119-120).

${ }^{15}$ Para un tratamiento de la diferencia entre "historia de la naturaleza" y "descripción de la naturaleza" en la evolución del pensamiento kantiano y en otros pensadores contemporáneos y posteriores, cf. Sloan (2006).

${ }^{16}$ En el "Prólogo" a MAN se afirma que el carácter científico en sentido propio que posee la física matemática depende de su parte pura (AA 04: 469 ss.) y de la aplicabilidad de la matemática en su ámbito (AA 04: 470-471).

${ }_{17}$ También la física matemática es sistemática. Sin embargo, el problema de en qué sentido este saber es sistemático no será tratado aquí, porque me ocupo de la sistematicidad sólo en la medida en que afecta al tipo de disciplina que contiene leyes empíricas científicas que emplean conceptos teóricos.
} 
ciencia. ${ }^{18}$ Las exigencias regulativas de introducción de conceptos teóricos y sistematización del conocimiento empírico están vinculadas entre sí, porque, por ejemplo, las leyes que emplean conceptos teóricos, a diferencia de las generalizaciones inductivas, son precisas y económicas y pueden ser integradas en sistemas estrictamente coherentes. ${ }^{19}$ Volveré a tratar en la Sección 2 el problema de las relaciones entre las dos exigencias mencionadas. ${ }^{20}$ Por otro lado, el examen del problema del tipo de ciencia tenido en cuenta en el AtD llevado a cabo hasta el momento nos permite formular dos conclusiones. En primer lugar, esta clase de disciplina esuna ciencia en un sentido impropio del término. A diferencia de la física matemática, la ciencia en sentido impropio es ciencia únicamente en virtud de su carácter sistemático y experimental. En segundo lugar, estetipo de ciencia incluye, al menos, a la química. ${ }^{21}$ Adelanto que, por motivos de espacio, no puede llevarse acabo aquí el extenso análisis de textos que requiere la consideración del problema de si, según el pensamiento kantiano en su período crítico, esa clase de ciencia está integrada únicamente por la química o incluye también otras disciplinas.

Se expusieron las características de los conceptos teóricos, su vinculación con las leyes empíricas científicas y la clase de ciencia que requiere leyes de este tipo. De acuerdo con los pasajes

${ }^{18}$ Según Friedman, MAN muestra cómo el ámbito de lo propiamente empírico es fundamentado determinadamente en el concepto trascendental de una naturaleza en general. La aplicación de los principios trascendentales del entendimiento al concepto empírico de materia consigue fundamentar la ley empírica newtoniana de gravitación universal (Friedman, 1992: 263). No dispongo aquí de espacio para evaluar esta interpretación. (Véanse las lecturas diferentes de Buchdahl 1965: 202 y 207-208, 1971: 34-44; Butts 1986: 190 ss. y Wartenberg 1979: 412-413, 1992: 240-241. Kant presenta en AtD1 el ejemplo de sistematización de las leyes del movimiento de los cuerpos celestes, y alude brevemente allí a la fuerza gravitacional como causa de ellas. Cf. KvR, A662 663/B690-691. Sin embargo, no estoy al tanto de base textual suficiente para valorar el señalamiento de Buchdahl y Butts de que la fuerza gravitacional es un concepto teórico del mismo orden que los demás. Cf. Buchdahl 1967: 217, 1992: 258 y 264-265 y Butts, 1986: 190 ss.). De acuerdo con Friedman, ciencias no matemáticas como la química de Stahl y la totalidad de la parte puramente experimental de la física no son comprendidas por la fundamentación de MAN (Friedman 1992: 174 ss., 240-242, 250 ss., 263-264 y 266-267). Asimismo, Friedman indica que, en el siglo XVIII, esas disciplinas pertenecientes a la "física experimental" se distinguían de las pertenecientes a la "física matemática", a saber, la mecánica racional, la óptica, la astronomía y la teoría newtoniana de la gravitación universal (Friedman 1992: 239, n. 38). Por último, Friedman estima que una de las motivaciones de Opus Postumum $(O P)$ es proveer una fundamentación para disciplinas incipientes del primer tipo como las ciencias del calor, la luz, la electricidad y el magnetismo, así como la química de Lavoisier (Friedman 1992: 263-264. Friedman observa que Kan habría adoptado la química de Lavoisier a más tardar en 1795. Ibíd.: 289). Como indicaré más adelante, no hay espacio aquí para examinar el problema de si en $\mathrm{KrV}$ o MAN Kant considera la química aisladamente o como perteneciente al conjunto de disciplinas que Friedman denomina "física experimental".

${ }^{19}$ Las generalizaciones de los fenómenos sustentadas en la observación casual efectuada hasta un cierto momento podrían denominarse leyes (en sentido amplio) empíricas ordinarias. Una de estas generalizaciones inductivas es el enunciado "el frío intenso congela el agua". Estos enunciados no son leyes en sentido estricto porque de ninguna manera pueden tenerse por necesarios. La experiencia ordinaria no implica necesariamente ni una coherencia ni una conexión mutua entre las diversas generalizaciones inductivas. Por tanto, esas generalizaciones no pueden ser integradas en sistemas estrictamente coherentes. Acerca de la necesidad "regulativa" de las leyes empíricas científicas, cf. infra, n. 19 y n. 46.

${ }^{20}$ Más arriba mostré cómo los conceptos y objetos teóricos hacen posibles explicaciones económicas y precisas de ciertas clases de fenómenos. En virtud de esta circunstancia, Brittan sostiene que las leyes que se refieren a objetos teóricos pueden ser tenidas por necesarias, al menos en tanto hayan sido verificadas (Brittan 1992: 176-177). Coincido parcialmente con Brittan, porque, como acabo de señalar, estimo que esas leyes también deben poder ser integradas en un sistema a fin de poder adquirir una necesidad que se podría llamar "regulativa" (cf. infra, n. 46). Para Brittan, en cambio, la sistematicidad es solamente una garantía de que nuestras teorías se refieren a un mundo único (Brittan 1992: 177-178).

${ }^{21}$ Como ya mencioné, en AtD1 Kant también hace referencia en una ocasión a las leyes del movimiento de los cuerpos celestes (cf. supra, n. 17). En lo que hace a la doctrina empírica del alma o psicología empírica, Kant afirma en MAN que ella no puede ser una ciencia, sino sólo una "descripción natural del alma" perteneciente a la doctrina histórica de la naturaleza. En primer lugar, esta psicología no es una ciencia en sentido propio porque la matemática no puede aplicarse adecuadamente a los fenómenos de sentido interno (acerca del sentido en el que la matemática no es aplicable de manera adecuada ni a la química ni a la psicología, cf. Nayak y Sotnak 1995). En segundo lugar, en la psicología empírica, a diferencia de la química, no es posible la experimentación ni la observación rigurosa: los fenómenos observados no pueden mantenerse separados ni ser recombinados a voluntad, no es posible someter a otro sujeto pensante a experimentos y la observación misma altera el estado de lo observado. Por tanto, esta psicología tampoco es una ciencia en sentido impropio como la química. Ciertamente, esa psicología puede aspirar a una cierta sistematicidad (MAN, AA 04: 471). Sin embargo, esta sistematicidad es puramente descriptiva y no conduce entonces a la formación de sistemas jerárquicos explicativos de leyes empíricas científicas. Estas leyes —esto es, las que emplean conceptos teóricos - deben ser puestas a prueba mediante experimentos, y no hay lugar para ellos en el ámbito de la psicología empírica. Por otro lado, en AtD1 Kant afirma que se debe intentar reducir las fuerzas de la mente (sensación, imaginación, memoria, etc.) a una única fuerza fundamental (A648-649/B676-677). En caso de que esta fuerza fundamental sea un concepto teórico, éste sería de una clase diferente de los utilizados en la química, puesto que, según lo dicho, no sería posible establecer una cierta validez objetiva de mismo mediante experimentación ni observación rigurosa. Sin embargo, estimo que la concepción kantiana de esa psicología en el AtD se revela cuando, en AtD2, se introduce la función regulativa de la idea del alma en tanto ente de razón. En virtud de esta función regulativa es posible una sistematización descriptiva de los fenómenos internos (KrV, A672/B700 y A682-684/B710-712). Acerca de las mencionadas tesis del AtD, cf. Sección 2 
citados y comentados, la doctrina kantiana de los conceptos teóricos podría interpretarse como una metodología del conocimiento empírico científico o una metateoría de la ciencia empírica fundada en una mera conveniencia subjetiva. Sin embargo, en un pasaje del AtD1 en el que se exponen los tres principios o leyes sistematizadores de la razón que presentaré en la Sección 2, Kant hace observaciones que considero válidas para la función regulativa de la razón teórica en general. Las leyes mencionadas juzgan que la "economía de las causas fundamentales" y otros requerimientos sistematizadores del conocimiento empírico "son [todos] en sí mismos conformes a la razón y adecuados a la naturaleza, y que por consiguiente esos principios llevan consigo su propia [carta de] recomendación de manera directa, y no meramente como procedimientos del método" (KrV, A660661/B688-689). ${ }^{22}$ Más aún, Kant señala que esos tres principios 'lógicos' son fundamentados por principios 'trascendentales'. ${ }^{23}$

Ahora ofreceré una interpretación de esa pretensión de objetividad o adecuación a la naturaleza, e incluso de carácter trascendental, de la función regulativa de la razón teórica en el caso de su exigencia de introducir conceptos teóricos. Como se indicó anteriormente, los conceptos teóricos pueden adquirir cierta validez objetiva cuando, en primer lugar, los elementos puros u objetos teóricos que ellos representan son pensados como contenidos en objetos empíricos y, en segundo lugar, las hipótesis de leyes que los emplean son puestas a prueba empíricamente con éxito. Además, puede interpretarse que estos conceptos son 'transcendentales' en el sentido, por cierto amplio, de que son condiciones de posibilidad, no ya de la experiencia en general, sino de lo que se podría llamar una 'experiencia científica'. ${ }^{24}$

Al considerar la vinculación entre conceptos teóricos y objetos empíricos, se desprende además que los conceptos teóricos que se refieren a objetos teóricos pensados como contenidos en cierta(s) clase(s) de objetos empíricos pueden ser incluidos como predicados o, según la terminología kantiana, "conceptos parciales" o "notas discursivas", en el contenido lógico del (de los) concepto(s) empírico(s) de esa(s) clase(s) de objetos empíricos. ${ }^{25}$ Podría pensarse, por ejemplo, que en los conceptos empíricos de nuestra experiencia externa ordinaria de objetos materiales están incluidos como conceptos parciales, si bien obscuramente, conceptos teóricos pertenecientes a una teoría simplificada de los elementos de la materia.

Cabe señalar que los conceptos empíricos, si bien no son condiciones de posibilidad de la experiencia de objetos empíricos en general, son por cierto condiciones de posibilidad de la experiencia de objetos empíricos en tanto objetos empíricos de ciertas clases. Por tanto, los conceptos teóricos, en la medida en que estén incluidos en los contenidos lógicos de los conceptos empíricos, pueden desempeñar el papel de condiciones de posibilidad de la experiencia en tanto que ella requiere conceptos empíricos, y ser entonces 'transcendentales' en un sentido amplio. ${ }^{26}$ Tal vez únicamente en este sentido tenga la exigencia regulativa de introducir conceptos teóricos una dimensión objetiva con respecto a la experiencia ordinaria. Sin embargo, tal exigencia regulativa no posee necesariamente esa

\footnotetext{
${ }^{22}$ La sistematización efectuada por la razón teórica no "es una operación meramente económica" de esa facultad. Tampoco es un "ensayo hipotético" fundamentado únicamente por su rendimiento de unidad en la explicación (KrV, A653/B681).

${ }^{23}$ Cf. KrV, A650-651/B678-679, A653-654/B681-682, A656-657/B684-685 y A660-661/B688-689.

${ }^{24}$ Hasta donde sé, Kant no usa la expresión "experiencia científica". Cf. infra, n. 26.

${ }^{25}$ El contenido lógico de un concepto es lo que se piensa en el mismo y está integrado por un concepto parcial o nota discursiva, o un conjunto de ellos. Los conceptos parciales o notas discursivas son representaciones universales y discursivas de propiedades que comparten todos los objetos que son casos particulares del concepto en cuestión. Ejemplos de notas discursivas contenidas en el concepto de cuerpo son la extensión, la impenetrabilidad y la figura ( KrV, B12. Sobre estas nociones, cf. Log, AA 09: 58 ss., 91 ss.).

Si bien hay un pasaje en el que Kant distingue las notas discursivas de los conceptos parciales (Log, AA 09: 95), estoy de acuerdo con Paton cuando mantiene que, para simplificar, es indicado desatender ese pasaje (Paton 1936/1970: I, 195-196). Kant mismo ignora la distinción mencionada en otros pasajes (cf. A43/B60 y Log, AA 09: 59-62).

${ }^{26} \mathrm{Mi}$ tesis es que la experiencia ordinaria puede envolver, y generalmente envuelve, el empleo de conceptos teóricos, aunque no necesariamente. Me opongo así, en primer lugar, a Brittan, quien sostiene que la experiencia ordinaria requiere conceptos teóricos para ser objetiva (Brittan 1992: 177). Además, mi tesis es diferente de la de Buchdahl, quien sostiene que, para Kant, el lenguaje observacional y el lenguaje teórico llevan vidas relativamente independientes (Buchdahl 1967: 222).
} 
dimensión objetiva, porque es perfectamente posible una experiencia ordinaria sin la intervención de conceptos teóricos. ${ }^{27}$

En síntesis, la exigencia regulativa de introducir conceptos teóricos tiene una dimensión objetiva y trascendental con respecto a una experiencia científica, y puede tener una dimensión objetiva y trascendental con respecto a la experiencia ordinaria. En lo que hace a este último punto, coincido parcialmente con una tesis de Peter Krausser. Aunque este último estima que su tesis es válida para la exigencia regulativa de sistematicidad, la presentaré aquí con referencia a la exigencia regulativa vinculada con los conceptos teóricos. Según Krausser, la función regulativa de la razón teórica puede entenderse como una versión temprana de la concepción, corriente hoy en día, de la carga teórica de la experiencia ordinaria o del carácter rector de lo teórico con respecto de tal experiencia. Esta función regulativa dirige la actividad real e histórica de construcción de sistemas de conceptos e hipótesis y, por tanto, de teorías. ${ }^{28}$ Por ende, gracias a esa función las teorías que en cierta época son tenidas por las mejores codeterminan inevitablemente y en gran parte lo que los objetos empíricos son para los sujetos de esa época. ${ }^{29}$ Disiento de Krausser en este último punto porque, como adelanté, entiendo que esa codeterminación no es inevitable, sino que, aunque usualmente efectuada, es en última instancia sólo posible.

Terminaré esta Sección con una indicación acerca del carácter indeterminado de las dimensiones objetiva y trascendental que acabo de presentar. En la "Analítica trascendental" de la primera Crítica (KrV, A64/B89 ss.) se intenta demostrar, entre otras cosas, que doce categorías son condiciones de posibilidad de la experiencia en general. Pero la exigencia regulativa de introducir conceptos teóricos carece de correlato objetivo o trascendental determinado. No es posible determinar ni a priori ni necesariamente qué conceptos teóricos adquirirán cierta validez objetiva y serán entonces condiciones de posibilidad de una experiencia científica, podrán ser así incluidos en los contenidos lógicos de los conceptos empíricos y desempeñar consiguientemente un papel como condiciones de posibilidad de la experiencia de objetos empíricos en tanto objetos empíricos de ciertas clases. Más aún, la exigencia regulativa de introducir conceptos teóricos, como se señaló, no es en sentido estricto necesaria para la experiencia mencionada en último lugar. Tal exigencia regulativa está hasta ahora justificada únicamente en la medida en que es necesaria para la posibilidad del tipo de ciencia presentado más arriba. Por tanto, su carácter necesario depende del carácter necesario de tal clase de ciencia. Esta cuestión será tratada más ampliamente en la Sección 2.

El estudio del uso hipotético regulativo de la razón y del requerimiento regulativo de sistematicidad que se efectuará en la Sección 2 permitirá, en primer lugar, completar la explicación del sentido en el que las leyes pertenecientes al tipo de ciencia consideradofundamentalmente en el AtD pueden adquirir una necesidad regulativa ${ }^{30} \mathrm{y}$, en segundo lugar, concluir la explicación del papel que desempeñan los conceptos empíricos como condiciones de posibilidad de la experiencia de objetos empíricos como objetos de ciertas clases. Se mostrará también que una de las funciones regulativas de la exigencia de sistematicidad es una condición de la aplicación del requerimiento regulativo de introducir conceptos teóricos. ${ }^{31}$

\footnotetext{
${ }^{27}$ Los conceptos de experiencia que mencioné, a saber, "experiencia científica" y "experiencia en tanto que requiere conceptos empíricos", son diferentes del concepto de experiencia que es decisivo en la "Analítica trascendental" (KrV, A64/B89 ss.), esto es, "enlace sintético de intuiciones empíricas de acuerdo con las categorías." Por tanto, las condiciones de posibilidad de los dos primeros tipos mencionados de experiencia son "trascendentales" en un sentido diferente del sentido en el que las condiciones de posibilidad de la experiencia en general lo son. Acerca de la indeterminación de las dos primeras condiciones de posibilidad, véase infra.

${ }^{28}$ Krausser remite en este punto a $\mathrm{KrV}$, A834/B862.

${ }^{29}$ Cf. Krausser 1987: 183-184.

${ }^{30}$ Cf. supra, n. 9 y Sección 2 (esp. n. 46).

${ }^{31}$ La lectura de McFarland en su conjunto, en cambio, coincide en general con los resultados de mi exposición hasta este momento. Según McFarland, lo regulativo es una condición del conocimiento científico y sólo puede mostrarse que, si no es presupuesto, la investigación científica se torna imposible (McFarland 1970: 18, 22-23, 29, 36-37, 42 y 88).
} 
2. El uso hipotético regulativo de la razón y el requerimiento regulativo de sistematicidad. Sus exigencias metodológicas y su dimensión objetiva

Algunas líneas después del pasaje en el que introduce la noción de concepto teórico, pasaje que comenté al inicio de la primera Sección, Kant presenta los conceptos de uso apodíctico y uso hipotético de la razón. Según el uso apodíctico de la razón, se debe partir de una regla universal que esté dada y sea cierta, a fin de determinar necesariamente los casos particulares que le corresponden. Pero existe también un uso de la razón diferente, esto es, el hipotético:

lo universal es supuesto sólo problemáticamente, y es una mera idea; lo particular es cierto, pero la universalidad de la regla para esa consecuencia es todavía un problema; entonces, varios casos particulares, que son todos ciertos, se ensayan con respecto a la regla [para ver] si se siguen de ella; y en ese caso, si parece ser que todos los casos particulares que puedan darse se siguen de ella, se infiere la universalidad de la regla, y a partir de ésta, después, se infieren también todos los casos que en sí mismos no están dados. (KrV, A646-647/B674-B675).

El uso hipotético de la razón presupone la existencia constatada de ciertos casos particulares. Se supone problemáticamente una ley empírica universal de la cual estos casos podrían seguirse, a fin de explicarlos en tanto consecuencias de la misma. Según Kant, los juicios problemáticos son formulados con la conciencia de su mera posibilidad, a saber, sin ser tenidos por verdaderos ni falsos, y su condición principal es la ausencia de contradicción. ${ }^{32}$ Por otro lado, al inicio del pasaje citado se afirma que lo universal supuesto problemáticamente "es una mera idea". Tal expresión indica, a mi parecer, que la ley empírica universal, postulada según el uso hipotético de la razón, debe emplear uno o más conceptos teóricos. ${ }^{33}$ Por tanto, puede afirmarse que este uso hipotético entraña la consideración inicial de hipótesis de leyes empíricas científicas como meramente posibles. $^{34}$

Krausser mostró convincentemente que en los textos kantianos pueden encontrarse cinco criterios según los cuales las hipótesis empíricas científicas deben ser admitidas y evaluadas. A diferencia de la interpretación aquí expuesta, Krausser considera esas hipótesis en tanto que emplean principalmente conceptos empíricos. Aquí sólo puede presentarse un resumen de tales criterios. 1) El objeto postulado por la hipótesis como fundamento de explicación no debe estar en desacuerdo con las condiciones de la experiencia. ${ }^{35}$ 2) El fundamento de explicación postulado por una hipótesis debe estar conectado con fenómenos dados y según al menos una ley empírica. ${ }^{36}$ 3) Debe ser posible deducir a priori los casos particulares dados de una hipótesis a partir de esa sola hipótesis. ${ }^{37}$ 4) Debe ser posible deducir a priori a partir de una hipótesis casos particulares que no estaban dados cuando la hipótesis fue formulada. ${ }^{38} 5$ ) Una hipótesis no debe precisar el apoyo de hipótesis auxiliares (esto es, ad hoc). En caso de que las precise, debe emplear la menor cantidad posible de ellas. ${ }^{39}$ Aunque Krausser considera casi exclusivamente las hipótesis que emplean sólo conceptos empíricos, aquí nos

\footnotetext{
32 "El mundo puede existir por ciego azar" es un ejemplo de juicio problemático (cf. KrV, A74-75/B100-101 y Log, AA 09: 108 109).

${ }^{33}$ Según Rajiva, la expresión "mera idea" puede referirse a los aquí llamados "conceptos teóricos" o al principio regulativo de sistematicidad de la razón (Rajiva 2006: 117-119). Aquí es preciso subrayar que el uso hipotético de la razón se aplica, o al menos puede aplicarse, a hipótesis que emplean conceptos teóricos. El hecho de que la expresión "mera idea" puede referirse a conceptos teóricos queda establecido porque la afirmación, según la cual en el uso hipotético de la razón lo "universal es supuesto sólo problemáticamente, y es una mera idea", se encuentra en el texto kantiano inmediatamente después del pasaje en el que se introduce la noción de "concepto teórico" (KrV, A646/B674).

${ }^{34}$ Presenté el tipo de ciencia del cual se ocupa principalmente el AtD en la Sección 1.

${ }^{35}$ Cf. KrV, A770-771/B798-799 y Log, AA 09: 85 .

${ }^{36} \mathrm{Cf}$. KrV, A770-B798 y A772/B800.

${ }^{37}$ Cf. KrV, B115, A774/B802 y Log, AA 09: 84-85

${ }^{38}$ Cf. KrV, A646-647/B674-675, A790-791/B818-819 y Log, AA 09: 84-85.

${ }^{39}$ Cf. KrV, B115, A774/B802 y Log, AA 09: 85-86. Para una explicación de estos cinco criterios, cf. Krausser (1987: 167-178) y Santos García (2004: 207-208).
} 
interesa únicamente la aplicación de estos criterios a las hipótesis que también emplean conceptos teóricos, ya que estas últimas son las consideradas principalmente en el AtD. Para aplicar esos criterios a estas hipótesis sólo es necesario reformular el primer criterio de la siguiente manera: el objeto teórico postulado por la hipótesis como fundamento de explicación debe ser pensado como contenido en objetos empíricos que estén de acuerdo con las condiciones de la experiencia. ${ }^{40}$

Las tres últimas exigencias metodológicas no son sólo criterios de admisión de hipótesis, sino también criterios de valoración comparativa de las mismas. Mientras menos hipótesis auxiliares necesite una hipótesis, más probable será; mientras más casos dados o no dados al momento de formularse la hipótesis puedan deducirse directamente de ella, más probable será (cf. Log, AA 09: 85). Sin embargo, aunque una hipótesis pueda tornarse más probable, ella nunca alcanzará una certeza apodíctica. A fin de concluir que una hipótesis es una verdad universal apodícticamente cierta sería necesario tener conocimiento de la verdad de todas las posibles consecuencias de esa hipótesis. Como esto no es posible en virtud de nuestra finitud, empleamos una inferencia analógica y afirmamos que, si todas las consecuencias de la hipótesis que hasta ahora conocemos son verdaderas, también serán verdaderas todas las restantes consecuencias posibles de ella. Por tanto, no es posible alcanzar una "certeza apodíctica" acerca de una hipótesis, sino tan sólo un "análogo de la certeza" [Analogon der Gewißheit] (Log, AA 09: 84-85). ${ }^{41} \mathrm{Al}$ contrario, la extracción de una sola consecuencia falsa a partir de una hipótesis basta para considerar esta última como falsa (KrV, A791/B819).

De todas maneras, las mencionadas tres últimas exigencias del uso hipotético regulativo de la razón, en tanto que conducen a la explicación de una o más clases de fenómenos dados y no dados a partir de una hipótesis de ley única, introducen un requerimiento de economía o simplicidad en la explicación científica. Este requerimiento también está presente, como se vio en la Sección 1, en la exigencia regulativa de introducir conceptos teóricos. Estas exigencias son entonces conjuntamente condiciones de la aplicación del principio regulativo de unidad sistemática de la razón teórica, dado que establecen una tendencia a la unidad en la explicación de la multiplicidad de los fenómenos. A continuación se expondrá el mencionado principio regulativo de unidad sistemática.

Al inicio de AtD1 se brinda una caracterización de las ideas de la razón que es válida fundamentalmente para estas ideas en tanto principios (los de homogeneidad, especificación y continuidad, expuestos en AtD1 $)^{42}$ y en tanto entes de razón (los del alma, el mundo y Dios, expuestos en AtD2). ${ }^{43}$ Estas dos clases de ideas están relacionadas con la exigencia de unidad sistemática de la razón teórica. Esta razón emplea regulativamente las mencionadas ideas para establecer la exigencia de conseguir la unidad y extensión sistemáticas absolutas de los conocimientos empíricos. Pero como un sistema absoluto de tales conocimientos sólo puede darse parcialmente en nuestra experiencia finita, este sistema puede únicamente desempeñar el papel de meta inalcanzable para todas las posibles acciones empíricas del entendimiento. De esta manera, la función regulativa de la razón teórica impone al entendimiento, como tarea necesaria, la consecución asintótica de la unidad y extensión sistemáticas completas del conocimiento empírico (cf. KrV, A642/B670 ss.).

La exigencia regulativa de sistematicidad lleva a la formación de diferentes clases de sistemas de conocimientos empíricos. Por un lado, están los sistemas clasificatorios jerárquicos de conceptos teóricos y empíricos. Por el otro, está cuando menos el sistema explicativo jerárquico de leyes empíricas científicas. ${ }^{44}$ Quisiera señalar que estos sistemas son interdependientes. En primer

${ }^{40}$ Acerca de las leyes científicas que emplean conceptos teóricos, véase la Sección 1. Sobre la física matemática, su parte pura y su carácter sistemático, véase también la Sección 1. Acerca de las generalizaciones inductivas, cf. supra, n. 18.

${ }^{41}$ Cf. KrV, A646-647/B674-675 y A790-791/B818-819.

${ }^{42}$ Cf. KrV, A657-658/B685-686 y A664-666/B692-694

${ }^{43} \mathrm{Cf} . \mathrm{KrV}, \mathrm{A} 670-673 / \mathrm{B} 698-701$

${ }^{44}$ Como adelanté, el problema de las leyes empíricas ordinarias en su especificidad no será examinado en profundidad en este trabajo. Tampoco me ocupo aquí del problema de las leyes pertenecientes a la física matemática. Cf. supra, n. 16, n. 17, n. 18, n. 39 e infra, n. 46 
lugar, para formular leyes empíricas científicas se deben poseer conceptos de los objetos teóricos y empíricos a los cuales ellas se refieren. En segundo lugar, las propiedades causales que las leyes empíricas científicas atribuyen a los objetos teóricos y empíricos se incluyen en los contenidos de los conceptos teóricos y pueden incluirse en los de los conceptos empíricos. ${ }^{45}$

La conexión entre la exigencia regulativa de sistematización y el uso hipotético regulativo de la razón se pone de manifiesto, según entiendo, en A647/B675. Allí se afirma: "[e]1 uso hipotético de la razón se dirige, pues, a la unidad sistemática de los conocimientos propios del entendimiento; y ésta, a su vez, es la piedra de toque de la verdad de las reglas". Estimo que, según la primera oración citada, el uso hipotético de la razón, tal como selo expuso hasta ahora, conduce a la aplicación del principio regulativo de unidad sistemática o, como se dijo antes, es una condición de esta aplicación. La segunda oración citada, a mi parecer, puede interpretarse en el sentido de que la posibilidad de integrar un concepto o una hipótesis de ley en el sistema correspondiente es uno de los criterios que se deben tener en cuenta para determinar la adecuación del concepto o el valor de verdad de la hipótesis de ley. ${ }^{46}$ Si bien se señaló que una hipótesis no puede ser verdadera en el sentido de alcanzar certeza apodíctica, la integrabilidad en un sistema de una hipótesis de ley que emplee conceptos teóricos y haya sido puesta a prueba empíricamente con éxito permite atribuirle a ella una necesidad que se podría llamar 'regulativa'. Puede afirmarse que la exigencia regulativa de sistematicidad, en la medida en que se ejerce sobre hipótesis de leyes, es un criterio más para la evaluación de las mismas, aunque presupone los cinco anteriores y es más complejo que ellos. ${ }^{47}$

La complejidad de la exigencia regulativa de sistematicidad consiste en que su ejercicio presupone necesariamente el empleo de las ideas de la razón en tanto principios y en tanto entes de razón. A continuación expondré estas clases de ideas en el orden en el que las mencioné. Presentaré entonces, en primer lugar, las ideas como principios, muy sucintamente y, por motivos de espacio, únicamente en tanto que conducen a la formación de sistemas de conceptos.

El principio de homogeneidad establece la necesidad de buscar la identidad de la especie en los diversos objetos empíricos singulares y la identidad del género en diferentes conceptos específicos

${ }^{45}$ Cf. las discusiones de Allison y Geiger (Allison 2001: 31; Geiger 2003: 276). Cf. también KrV, A664/B692.

${ }^{46}$ Cf. KrV, A651/B679; Krausser (1987: 180-181 y 184-185); Santos García (2004: 207) y (Abela 2006: 419 ss.).

47 "Incluso las leyes de la naturaleza, si se las considera como principios del uso empírico del entendimiento, llevan consigo, a la vez, una expresión de necesidad, y por tanto, al menos la sospecha de una determinación a partir de fundamentos que son válidos $a$ priori y antes de toda experiencia. Pero todas las leyes de la naturaleza, sin diferencia, están sometidas a principios superiores de entendimiento, pues ellas sólo los aplican a éstos a casos particulares del fenómeno" (KrV, A159/B198. Cf. KrV, B165 y A216/B263). "Esta idea [MA: de una totalidad del conocimiento] postula, según eso, una unidad completa del conocimiento que es propio del entendimiento, en virtud de la cual ese [conocimiento] no se constituye como un mero agregado contingente, sino que llega a ser un sistema interconectado según leyes necesarias" (KrV, A645/B673). El segundo pasaje citado puede interpretarse en el sentido de que sólo las leyes empíricas científicas que formen parte del sistema total del conocimiento empírico podrían ser consideradas como necesarias. Este sistema total, como señalé, es una meta inalcanzable que orienta necesariamente al accionar de entendimiento. Una ley de esa clase, esto es, que emplea conceptos teóricos, puede ser tenida por necesaria en caso de que sea posible integrarla en un sistema de esa clase de leyes que no haya sido falsado, porque así es posible suponer que esa ley podrí llegar a ser incluida en el sistema completo del conocimiento empírico. No obstante, el sistema de leyes del cual la ley en cuestión pasaría a formar parte podría ser falsado en el futuro, o ser remplazado por otro sistema que provea más explicaciones o explicaciones más precisas. Por tanto, en última instancia, las leyes empíricas científicas no pueden adquirir, en nuestra experiencia finita, más que lo que se podría denominar un "análogo de necesidad". Esta necesidad puede llamarse "regulativa", porque sólo puede ser alcanzada, en sentido estricto, en el marco del sistema total del conocimiento empírico que la función regulativa de la razón teórica impone como meta que se debe perseguir asintóticamente. Las leyes empíricas científicas pueden alcanzar grados de precisión, simplicidad y potencial explicativo considerablemente mayores que los de las generalizaciones inductivas, porque las primeras emplean conceptos teóricos y son integrables en sistemas estrictamente coherentes. La experiencia ordinaria, por el contrario, no implica necesariamente ni una coherencia ni una conexión estrictas entre las diversas generalizaciones inductivas. Por tanto, estas generalizaciones ni siquiera pueden ser supuestas como pertenecientes al sistema completo del conocimiento empírico Cf. supra, n. 16, n. 17, n. 18, n. 19 y n. 39. Coincido, entonces, con Buchdahl cuando sostiene que el principio de sistematicidad y el requerimiento de introducir los aquí denominados "conceptos teóricos" son conjuntamente condiciones de una cierta necesida de las leyes empíricas científicas que se podría llamar "empírica" o "regulativa". Sin embargo, Buchdahl no expone en detalle las propiedades de esas dos prescripciones metodológicas y de sus interrelaciones, y enfatiza unilateralmente la exigencia de sistematicidad (cf. Buchdahl 1965, 1967 y 1969). Otras discusiones enfatizan exclusivamente o aún más unilateralmente la conexión entre la exigencia de sistematicidad y una cierta necesidad de las leyes empíricas (cf. Buchdahl 1971; Kitcher 1986 y Krausser 1987). Por último, otros intérpretes enfatizan unilateralmente la exigencia regulativa de introducir conceptos teóricos (cf. Brittan 1992 y McNulty 2015). La compleja interpretación de McNulty no puede analizarse aquí en detalle por motivos de espacio. 
teóricos y empíricos (KrV, A651-654/B679-682). ${ }^{48}$ La aplicación de este principio conduce así a la máxima 'unidad' sistemática posible de los conceptos teóricos y empíricos. Debe notarse que la búsqueda de identidad entre conceptos de órdenes cada vez más elevados puede detenerse en caso de que se alcance un género supremo, esto es, un concepto que ya no pueda ser entendido como una especie de otro concepto más genérico.

El principio de especificación plantea el requerimiento de buscar la división de cada género en especies diferentes y de cada especie en subespecies diferentes (KrV, A654-657/B682-685). Siempre es posible encontrar entre los casos particulares de un cierto concepto, además de las propiedades comunes a todos ellos (propiedades cuyas representaciones discursivas y universales constituyen el contenido de ese concepto), conjuntos de diferencias significativas que permitan, por ejemplo, conceder validez objetiva a dos posibles especies de tal concepto. ${ }^{49}$ Por tanto, el principio de especificación puede aplicarse indefinidamente y conduce así a la máxima 'extensión' sistemática posible de los conceptos teóricos y empíricos. ${ }^{50}$

El principio de continuidad conecta las exigencias de máxima unidad genérica y máxima extensión de contenido, porque establece el requerimiento de que, entre dos especies de un mismo género que se consideren inicialmente como colindantes, se introduzcan cada vez más especies intermedias. Una especie intermedia introducida de esta manera es diferente de la primera y de la segunda en menor medida que estas dos últimas entre sí. El principio de continuidad, por tanto, tiende a la producción de un tránsito continuo entre las diversas especies de cada uno de los géneros $(\mathrm{KrV}$, A657-661/B685-689). ${ }^{51}$ Este principio puede aplicarse indefinidamente, porque, según Kant, desde el punto de vista de la razón no puede haber especies "inmediatas unas a otras" (KrV, A659/B687).

El concepto posible que expresa la identidad de contenido de dos o más conceptos del mismo orden (homogeneidad), la división del contenido de un concepto (especificación) o la continuidad relativa entre especies de un concepto (continuidad) es creado por la razón teórica. Tal concepto permanece posible hasta que el entendimiento, así orientado por la razón y en vinculación con la sensibilidad, pueda concederle ciertavalidez objetiva mediante mera observación (concepto empírico) o experimentación (concepto teórico). ${ }^{52}$ En A643/B671 Kant afirma: "[1]a razón no se refiere nunca directamente a un objeto, sino solamente al entendimiento [...] [pues] ella no crea conceptos (de objetos), sino que solamente los ordena". Aunque este pasaje parezca contradecir la tesis según la cual la razón puede crear conceptos de objetos, estimo que esta facultad, al ejercer su actividad sistematizadora, crea conceptos posibles a fin de rellenar los 'huecos' que quedan en el sistema de los conceptos de objetos existentes y establecer así relaciones entre estos últimos conceptos. Como solamente el entendimiento, vinculado con la sensibilidad, puede conceder validez

\footnotetext{
${ }^{48}$ Dado que los conceptos y objetos teóricos son postulados conjuntamente por la razón, no puede haber objetos teóricos sin conceptos teóricos que les correspondan. Por otro lado, véanse los ejemplos de aplicación del principio de homogeneidad en el ámbito de la química en KrV, A652-653/B680-681. Allí afirma Kant, por ejemplo, que los químicos redujeron "todas las sales a dos géneros principales, las ácidas y las alcalinas" e intentaron también reducir esta diversidad a un género único.

${ }^{49}$ Según la razón, ninguna especie puede considerarse "como la [especie] ínfima" (KrV, A655/B683). Por otro lado, a fin de encontrar conjuntos de diferencias significativas entre los casos particulares de un concepto teórico, no deben examinarse únicamente los correspondientes objetos teóricos contenidos en diversos objetos empíricos, porque esos objetos teóricos son, por definición, idénticos. Es preciso examinar también los objetos empíricos en los cuales esos objetos teóricos están contenidos. Tales objetos empíricos deben ser estudiados solamente en la medida en que contienen los objetos teóricos relevantes, y a fin de obtener indicios acerca de los mencionados conjuntos de diferencias significativas.

${ }^{50}$ Véase el ejemplo perteneciente a la química presentado por Kant en KrV, A657/B685. Allí se menciona allí el descubrimiento de que las tierras absorbentes son de dos especies (tierras calcáreas y muriáticas).

${ }^{51}$ Kant presenta el ejemplo de aplicación del principio de continuidad a las leyes del movimiento de los cuerpos celestes ( KrV A662-663/B690-691). Goldberg propone un ejemplo de aplicación de este principio a los conceptos aquí llamados teóricos teniendo en cuenta un estado de la ciencia posterior al de la época de Kant. Si la mente poseyera los conceptos de carbono-12 y carbono-14, la aplicación del mencionado principio llevaría a la producción del concepto posible de carbono-13 (cf. Goldberg 2004: 407 e infra).

${ }^{52}$ Coincido en este punto con Goldberg y Rauscher, aunque Goldberg no distingue entre conceptos teóricos y empíricos y Rauscher no enfatiza suficientemente esta distinción (cf. Goldberg 2004: 407 y 419 y Rauscher 2010: 295-297).
} 
objetiva a esos conceptos posibles, esta interpretación no contradice la tesis kantiana según la cual la razón no se refiere nunca 'directamente' a un objeto, sino al entendimiento.

Anteriormente se mostró que, en general, la exigencia regulativa de introducir conceptos teóricos es una condición de la aplicación del principio regulativo de unidad sistemática de la razón, porque establece una tendencia a la unidad en la explicación de los fenómenos. El análisis del principio regulativo de sistematicidad permite concluir, por otro lado, que este principio puede llevar al diseño de novedosos experimentos que ponen a prueba hipótesis que emplean conceptos teóricos.

En AtD2 Kant presenta una tríada diferente de ideas de la razón, a saber, la de las ideas como 'entes de razón'. Tales entes de razón no son objetos existentes, sino el contenido temático de las ideas en tanto conceptos, considerado como un objeto o, tal vez más exactamente, cuasi-objeto. Hay tres de estos entes de razón: los del alma, el mundo y Dios. ${ }^{53}$ Según Kant, podemos y tenemos que representar de manera sistemática los objetos empíricos en la medida en que los referimos a tales entes de razón.

La concepción de las ideas de AtD2 y de su relación con la de AtD1 presentan una complejidad que las hace merecedoras de un estudio independiente. Aquí me limitaré a resumir las conclusiones al respecto que alcancé en un trabajo previo. ${ }^{54}$ En ese trabajo coincidí con Caimi y Allison en que las concepciones de las ideas de AtD1 y AtD2 pueden integrarse en una teoría coherente, en la medida en que las ideas como entes de razón se entienden como condiciones de la aplicación de las ideas como principios. Además, procuré poner al descubierto los modos en los que las ideas como entes de razón orientan y determinan la aplicación de las ideas como principios. ${ }^{55}$

Las ideas presentadas en AtD2, a diferencia de las ideas como principios, no se aplican a la totalidad de los conceptos empíricos y teóricos y de las leyes empíricas científicas que los emplean, sino a los fenómenos y desde un determinado punto de vista. ${ }^{56}$ El ente de razón de la idea de alma hace necesario alcanzar la más completa unidad sistemática posible de las determinaciones de la mente, en la medida en que estas últimas se conciben como potencias o cambios de estado de la misma substancia simple y permanente y, entonces, como diferentes de los fenómenos externos. ${ }^{57} \mathrm{La}$ referencia de todos los fenómenos al ente de razón de la idea de mundo hace necesario que en la explicación de ellos (esto es, en la conceptualización de los mismos y su sometimiento a leyes empíricas) se proceda como si sus series de estados de ellos fuesen infinitas. ${ }^{58}$ Por último, el ente de razón de la idea de Dios conduce necesariamente a concebir todo ordenamiento del mundo como si hubiera surgido del designio de una razón suprema y, por tanto, como conforme a fines [zweckmäßig]. Gracias a esta presuposición pueden hacerse descubrimientos de acuerdo con la causalidad mecánica que de otro modo no hubieran sido notados. ${ }^{59}$

Mencionaré dos ejemplos de determinación de la aplicación de las ideas como principios mediante las ideas como entes de razón. Como las ideas en tanto principios contienen ya en sí mismas una exigencia de sistematización, podría preguntase, por ejemplo, en qué medida la aplicación de estos principios a los fenómenos del sentido interno requiere como su condición el empleo del ente de razón de la idea del alma. El punto es que las exigencias de sistematización de las ideas como principios no implican necesariamente que pueda encontrarse una unidad sistemática de la

${ }^{53}$ Una caracterización de las ideas como entes de razón es ofrecida por Caimi (cf. Caimi 1996: 76 ss.). La expresión "cuasi-objeto" [Quasi-Gegenstand] es introducida por Zocher (cf. Zocher 1958: 48)

${ }^{54}$ Cf. Arias Albisu 2012

${ }^{55}$ Cf. Caimi 1995: 319 y Allison 2004: 438-439. Morrison presenta una tesis similar (cf. Morrison 1989: 164-166). La divergencia entre las concepciones de las ideas de AtD1 y AtD2 es subrayada por Zocher (cf. Zocher 1958: 58 y 1966: 225).

${ }^{56} \mathrm{Cf}$. KrV, A681/B709 y supra, n. 39. La sistematización de los fenómenos afecta a los conceptos empíricos y a los conceptos teóricos de elementos puros contenidos en esos fenómenos. Además, los sistemas de conceptos y de leyes son interdependientes.

${ }^{57} \mathrm{Cf} . \mathrm{KrV}, \mathrm{A} 672 / \mathrm{B} 700$ y A682-684/B710-712.

${ }^{58}$ Cf. KrV, A672/B700 y A684-685/B712-713.

${ }^{59}$ Cf. KrV, A672-673/B700-701 y A685/B713 ss. 
experiencia interna. Esta exigencia ulterior de unidad sistemática de los fenómenos del sentido interno, que orienta la aplicación de las ideas como principios a estos fenómenos, es posible por el ente de razón de la idea del alma. Sin esta orientación ulterior, la aplicación del principio de homogeneidad podría conducir a la infructuosa tarea de buscar homogeneidad entre una facultad de la mente y un objeto en el espacio. Por otro lado, como no puede haber experimentación en el ámbito de la experiencia interna (cf. supra, n. 20), el empleo del ente de razón de la idea de alma tiene también la utilidad negativa de invalidar los intentos de formular hipótesis que empleen conceptos teóricos referidos a objetos teóricos contenidos en fenómenos internos que deban ser sometidas a experimentación.

Por su parte, el ente de razón de la idea de mundo exige que, para, por ejemplo, considerar una ley causal que explique ciertos cambios de estado, no apliquemos el principio de homogeneidad sólo a los cambios de estado de los fenómenos dados actualmente a los sentidos, sino que también examinemos cambios de estado documentados del pasado y continuemos estudiándolos en el futuro, sin terminar nunca esta búsqueda.

Concluiré esta Sección con una reflexión acerca de la dimensión objetiva y trascendental de las exigencias metodológicas del uso hipotético regulativo de la razón y del principio regulativo de sistematicidad. Como se adelantó en la Sección 1, las tres ideas de la razón en tanto principios son, según Kant, principios lógicos o metodológicos que requieren una fundamentación provista por los tres principios trascendentales que les corresponden. Explicaré esta cuestión resumiendo las tesis de Caimi acerca de ella (cf. Caimi 1995: 311-317). Sería irracional e injustificado buscar la unidad sistemática de los conocimientos empíricos exigida por los principios lógicos de homogeneidad, especificación y continuidad, si no se presupusiera a priori que la mencionada unidad pertenece a los objetos empíricos mismos. Tal presuposición de sistematicidad "como perteneciente a los objetos mismos" (KrV, A650/B678) se torna necesaria en virtud de los principios trascendentales de homogeneidad, especificación y continuidad. Estos principios trascendentales, por su parte, no son legítimos porque sirvan de fundamento para los principios lógicos, dado que estos últimos podrían ser arbitrarios. Tales principios son trascendentales, en cambio, porque son condiciones de posibilidad de la experiencia de los objetos empíricos como objetos de ciertas clases. ${ }^{60}$ Por ejemplo, si no se presupusiera, de acuerdo con el principio trascendental de homogeneidad, que los objetos empíricos que tienen la misma apariencia tienen también la misma constitución interna, no sería posible abstraer conceptos empíricos a partir de tales objetos, ${ }^{61}$ y, como se señaló, sin conceptos empíricos no es posible la experiencia de objetos empíricos en tanto objetos empíricos de determinadas clases. ${ }^{62}$

La exigencia regulativa de sistematicidad tiene entonces una dimensión objetiva e incluso trascendental. Esta dimensión es indeterminada. Afirma Kant, por ejemplo, que de acuerdo con el principio trascendental de homogeneidad presuponemos necesariamente homogeneidad en lo múltiple de la experiencia, "aunque no podamos determinar a priori el grado de ella" (KrV, A654/B682). Esta afirmación significa que, aunque tenemos que presuponer a priori la unidad sistemática del conocimiento en la naturaleza misma, no podemos determinar a priori el grado de unidad sistemática que encontraremos en la naturaleza en un momento dado. ${ }^{63}$

La dimensión objetiva del requerimiento regulativo de sistematicidad que se acaba de exponer corresponde fundamentalmente a ese requisito en tanto que concierne a los conceptos

${ }^{60}$ Cf. KrV, A650-651/B678-679, A653-654/B681-682, A656-657/B684-685 y A660-661/B688-689.

${ }^{61}$ Cf. KrV, A653-654/B681-682 y EEKU, AA 20: 215-216.

${ }^{62}$ Acerca de los distintos conceptos de "experiencia" y "estatus trascendental", véase supra, n. 26. Dado que las ideas como entes de razón son condiciones de la aplicación de las ideas como principios, la función trascendental de las últimas requiere el e mpleo de las primeras.

${ }^{63}$ Cf. KrV, A653/B681, A665/B693 y A668/B696. Wartenberg entiende la distinción entre principios lógicos y trascendentales como una distinción entre principios metodológico-regulativos y el conocimiento a priori e indeterminado incorporado en los mismos (cf. Wartenberg 1979: 414-424). 
empíricos. Sin embargo, aquí me ocupo de las leyes empíricas más tenidas en cuenta, más bien, en el AtD, a saber, las leyes empíricas científicas que emplean conceptos teóricos. Con respecto a este último punto, mostraré, en primer lugar, que existe una conexión entre, por un lado, la exigencia regulativa de introducir conceptos teóricos y las tres últimas exigencias del uso hipotético regulativo de la razón y, por el otro, el principio regulativo de sistematicidad. En segundo lugar, expondré la conexión que existe entre los mencionados requisitos relacionados con los conceptos teóricos y el requisito de sistematicidad referido a los conceptos empíricos.

En la Sección 1 se mostró que las leyes empíricas científicas requieren el empleo de conceptos teóricos para explicar precisa y económicamente cierta(s) clase(s) de fenómenos. Además, al comienzo de la Sección presente se indicó que las tres últimas exigencias del uso hipotético regulativo de la razón tienden a introducir unidad en la explicación de los fenómenos. De este modo, estas prescripciones conducen a la formación de hipótesis de leyes que, en virtud de las características mencionadas y a diferencia de las generalizaciones inductivas, pueden ser integradas en sistemas de leyes estrictamente coherentes (cf. supra, n. 46). Como se señaló, el principio regulativo de sistematicidad exige la formación de sistemas cada vez más unificados y extensos de los conocimientos empíricos. Si dispusiéramos sólo de generalizaciones inductivas y no tuviéramos leyes con potencial explicativo amplio y económico, entonces el principio regulativo de sistematicidad no podría formar un sistema de leyes empíricas científicas que sea estrictamente coherente y además suministre una explicación económica de una gran variedad de fenómenos. ${ }^{64}$ Por otra parte, como se afirmó, la formación de este sistema y la formación de un sistema de conceptos teóricos son interdependientes.

Al final de la Sección 1 se vio que los conceptos teóricos son condiciones de posibilidad de lo que se podría llamar una 'experiencia científica'. Además, estos conceptos pueden ser condiciones de posibilidad de la experiencia de objetos empíricos como objetos empíricos de ciertas clases, en la medida en que tales conceptos teóricos sean incluidos como notas discursivas en el contenido lógico de conceptos empíricos. Sin embargo, como se indicó en la Sección 1, es posible que tenga lugar una experiencia ordinaria sin la intervención de conceptos teóricos. ${ }^{65}$ Las tesis sobre los conceptos teóricos expuestas en este párrafo valen también para las leyes empíricas científicas.

En conclusión, si bien la exigencia de introducir conceptos teóricos, las tres últimas exigencias del uso hipotético de la razón y la exigencia de sistematicidad referida a los conceptos teóricos y las leyes que los emplean conducen conjuntamente a la formación de un sistema de leyes empíricas científicas, tales exigencias no poseen una dimensión objetiva propia más amplia que la que acabo de exponer, a saber, la consistente en una experiencia científica.

Cabe señalar que estas exigencias vinculadas con los conceptos teóricos sólo pueden aplicarse bajo el presupuesto de la aplicación de la exigencia de sistematicidad referida a los conceptos empíricos. Anteriormente se mostró que la última exigencia es una condición de posibilidad de los conceptos empíricos. Y, ciertamente, no es posible formar, por ejemplo, un concepto teórico que represente un objeto teórico cuya función sea esclarecer los procesos de calcinación y combustión sin poseer previamente conceptos empíricos tales como los de plomo y de carbón. Sin tales conceptos empíricos, sería imposible identificar todos los objetos empíricos correspondientes a fin de formar el mencionado concepto teórico.

Por último, quisiera distinguir dos tesis presentadas en los dos últimos párrafos. Los conceptos empíricos hechos posibles por la exigencia de sistematicidad referida a ellos son condiciones de posibilidad de la formación de los conceptos teóricos. Una vez que se han formado

${ }^{64}$ Por otro lado, como ya indiqué, el requerimiento de sistematicidad puede conducir al diseño de experimentos que ponen a prueba hipótesis que utilizan conceptos teóricos.

${ }^{65}$ Cf. supra, n. 23, n. 24 y n. 26. 
tales conceptos teóricos, es posible incluirlos como notas discursivas en el contenido lógico de los conceptos empíricos.

\section{Conclusiones}

En la Sección 1 se estudió la exigencia regulativa de introducir conceptos teóricos. Mostré allí que la clase de disciplina considerada con más atención en el AtD, esto es, al menos, la química, ${ }^{66}$ está integrada por leyes empíricas científicas que se distinguen por su empleo de conceptos teóricos. Por otro lado, indiqué, en primer lugar, que la exigencia mencionada tiene una dimensión objetiva y trascendental con respecto a una experiencia científica. En segundo lugar, señalé que la exigencia en cuestión puede entenderse como condición de posibilidad de la experiencia de objetos empíricos como objetos empíricos de ciertas clases, en la medida en que los conceptos teóricos sean incluidos como notas discursivas en el contenido lógico de conceptos empíricos. Sin embargo, es posible que haya una experiencia que involucre conceptos empíricos que no posean como notas discursivas conceptos teóricos. Por último, mostré que la exigencia regulativa de introducir conceptos teóricos tiene una dimensión objetiva indeterminada. No puede establecerse a priori qué conceptos teóricos obtendrán cierta validez objetiva y serán así condiciones de posibilidad de una experiencia científica, ni tampoco cuáles de estos conceptos objetivamente válidos podrán ser integrados en los contenidos lógicos de conceptos empíricos y ser entonces condiciones de posibilidad de la experiencia de objetos empíricos como objetos empíricos de determinadas clases.

En la Sección 2 se mostró que la exigencia regulativa de introducir conceptos teóricos, las tres últimas exigencias del uso hipotético regulativo de la razón y la exigencia regulativa de sistematicidad referida a los conceptos teóricos y las leyes que los emplean son condiciones de posibilidad de la formación de un sistema de leyes empíricas científicas poseedoras de una necesidad 'regulativa', así como de la formación de un sistema de conceptos teóricos. Por tanto, estas tres exigencias mencionadas son conjuntamente condiciones de posibilidad de una experiencia científica. Además, pueden ser condiciones de posibilidad de la experiencia de objetos empíricos como objetos empíricos de ciertas clases en el sentido explicado en el párrafo anterior.

Otra conclusión importante de la Sección 2 es que el requerimiento regulativo de sistematicidad relativo a los conceptos empíricos es condición de posibilidad de estos conceptos. Esta dimensión objetiva de tal requerimiento es, como afirmé, también indeterminada. Además, los conceptos empíricos son condiciones de posibilidad de los conceptos teóricos. Considero que los aportes fundamentales del AtD acerca de la dimensión objetiva de la función regulativa de la razón teórica son los dos siguientes: i) la especificación de las condiciones que deben cumplir las leyes empíricas para adquirir la necesidad regulativa que las convierte en leyes científicas que pueden pertenecer, al menos, a la química. Estas condiciones son el empleo de conceptos teóricos y la integrabilidad en sistemas de leyes estrictamente coherentes y de un poder explicativo amplio, preciso y económico. Las leyes que poseen tal necesidad regulativa son condiciones de posibilidad de una experiencia científica y, como se vio, 'pueden' ser asimismo condiciones de posibilidad de la experiencia de objetos empíricos como objetos empíricos de ciertas clases. ii) La caracterización de la exigencia regulativa de sistematicidad relativa a los conceptos empíricos como condición de posibilidad de los conceptos empíricos y, por tanto, de la experiencia de objetos empíricos en tanto

${ }^{66}$ Como indiqué en la Sección 1, no dispongo de espacio para examinar el problema de si la clase de ciencia que expuse en este artículo está, de acuerdo con el pensamiento de Kant en el período crítico, integrada sólo por la química o incluye también otras disciplinas 
objetos empíricos de ciertas clases y, como también señalé, de los conceptos teóricos y las leyes que los emplean. ${ }^{67}$

Por último, estimo que mi exposición pone al descubierto la falencia más importante de las discusiones contenidas en el AtD, a saber, la ausencia de una fundamentación de la experiencia científica que vaya más allá del señalamiento según el cual las leyes empíricas científicas suministran explicaciones unificadas referidas a amplios conjuntos de fenómenos.

\section{Bibliografía}

ABELA, Paul: “The Demands of Systematicity: Rational Judgment and the Structure of Nature", en BIRD, G. (ed.): A Companion to Kant, Oxford/Malden, Blackwell, 2006.

ALLISON, Henry E.: Kant's Theory of Taste, Cambridge, Cambridge University Press, 2001.

ALLISON, Henry E.: Kant's Transcendental Idealism. Revised and Enlarged Edition, New Haven/London, Yale University Press, 2004.

ARIAS ALBISU, Martín: “Acerca de la relación entre los dos tipos de esquemas de las ideas de la razón en la Crítica de la razón pura de Kant”, Areté 24:1 (2012) 7-24.

ARIAS ALBISU, Martín: "Las prescripciones metodológicas de la función regulativa de la razón teórica en la 'Crítica de la razón pura'de Kant”, Kant e-Prints 10:1 (2015) 64-93.

BENNETT, Jonathan: Kant's Dialectic, Cambridge, Cambridge University Press, 1974.

BRITTAN, Gordon G.: "Systematicity and Objectivity in the Third Critique", The Southern Journal of Philosophy 30:S1 (1992) 167-186.

BUCHDAHL, Gerd: "Causality, Causal Laws and Scientific Theory in the Philosophy of Kant", The British Journal for the Philosophy of Science 16 (1965) 187-208.

BUCHDAHL, Gerd: "The Relation between 'Understanding' and 'Reason' in the Architectonic of Kant's Philosophy”, Proceedings of the Aristotelian Society 67 (1967) 209-226.

BUCHDAHL, Gerd: "The Kantian 'Dynamic of Reason', with Special Reference to the Place of Causality in Kant's System", en BECK, L. W. (ed.): Kant Studies Today, La Salle, Open Court, 1969.

BUCHDAHL, Gerd: “The Conception of Lawlikeness in Kant's Philosophy of Science”, Synthese 23 (1971) 24-46.

BUCHDAHL, Gerd: "Gravity and Intelligibility: Newton to Kant", en BUCHDAHL, G. (ed.): Kant and the Dynamics of Reason, Oxford, Blackwell, 1992.

BUTTS, Robert E.: "The Methodological Structure of Kant's Metaphysics of Science", en BUTTS, R. E. (ed.): Kant's Philosophy of Physical Science, Dordrecht, D. Reidel, 1986.

CAIMI, Mario: "Über eine wenig beachtete Deduktion der regulativen Ideen", Kant Studien 86 (1995) 308-320.

CAIMI, Mario: "La función regulativa del ideal de la razón pura”, Diánoia 42 (1996) 61-79.

CARRIER, Martin: "Kant's Theory of Matter and His Views on Chemistry”, en WATKINS, E. (ed.): Kant and the Sciences, Oxford, Oxford University Press, 2001.

FRIEDMAN, Michael: Kant and the Exact Sciences, Cambridge, Harvard University Press, 1992.

GEIGER, Ido: "Is the Assumption of a Systematic Whole of Empirical Concepts a Necessary Condition of Knowledge?", Kant Studien, 94 (2013) 273-298.

${ }^{67}$ Véase el último párrafo de la Sección 2. 
GOLDBERG, Nathaniel J.: "Do Principles of Reason Have 'Objective but Indeterminate Validity'?”, Kant Studien 95 (2004) 405-425.

KANT, Immanuel: Kant's gesammelte Schriften, Deutsche (anteriormente Königlich Preußische) Akademie der Wissenschaften (ed.), Berlin y otros, Walter de Gruyter y predecesores, 1900ss. (Akademie-Ausgabe=AA).

KANT, Immanuel: Lógica. Un manual de lecciones. Edición original de G. B. Jäsche, traducción castellana de M. J. Vázquez Lobeiras, Madrid, Akal, 2000 (Log).

KANT, Immanuel: Crítica de la razón pura, traducción castellana de M. Caimi, México, Fondo de Cultura Económica, UAM, UNAM, 2009 (KrV, A/B).

KANT, Immanuel: Primeros principios metafísicos de la ciencia de la naturaleza, traducción castellana de S. Nemirovsky, México, Universidad Nacional Autónoma de México, 1993 (MAN)

KEMP SMITH, Norman: A Commentary to Kant's “Critique of Pure Reason”. Second edition, revised and enlarged, New York, Palgrave Macmillan, 1923/2003.

KITCHER, Philip: "Projecting the Order of Nature", en BUTTS, R. E. (ed.): Kant's Philosophy of Physical Science, Dordrecht, D. Reidel, 1986.

KRAUSSER, Peter: "Über den hypothetischen Vernunftgebrauch in der Kritik der reinen Vernunft", Archiv für Geschichte der Philosophie 69 (1987) 164-196.

McFARLAND, John D.: Kant's Concept of Teleology, Edinburgh, University of Edinburgh Press, 1970.

McNULTY, Michael B.: "Rehabilitating the Regulative Use of Reason: Kant on Empirical and Chemical Laws", Studies in History and Philosophy of Science 54 (2015) 1-10.

MORRISON, Margaret: "Methodological Rules in Kant's Philosophy of Science", Kant Studien 80 (1989), 155-172.

NAYAK, Abhaya C. y SOTNAK, Eric: "Kant on the Impossibility of the "Soft Sciences", Philosophy and Phenomenological Research 55 (1995) 133-151.

PATON, Herbert J.: Kant's Metaphysic of Experience, London, George Allen \& Unwin Ltd., $1936 / 1970$.

RAJIVA, Suma: "Is Hypothetical Reason a Precursor to Reflective Judgment?", Kant Studien 97 (2006) 114-126.

RAUSCHER, Frederick: "The Appendix to the Dialectic and the Canon of Pure Reason. The Positive Role of Reason", en GUYER, P. (ed.): The Cambridge Companion to Kant's Critique of Pure Reason, Cambridge, Cambridge University Press, 2010.

SANTOS GARCÍA, Miguel Á.: "Kant y la lógica de la investigación científica”, Teorema 23 (2004) 199-213.

SLOAN, Philip R.: "Kant on the history of nature: The ambiguous heritage of the critical philosophy for natural history", Studies in History and Philosophy of Biological and Biomedical Sciences 37 (2006) 627-648.

STAHL, Georg E.: Zufällige Gedancken und nützliche Bedencken über den Streit von dem sogenannten Sulphure, Halle, Wäysenhaus, 1718.

WARTENBERG, Thomas E.: "Order through Reason. Kant's Transcendental Justification of Science", Kant Studien 70 (1979) 409-424.

WARTENBERG, Thomas E.: "Reason and the Practice of Science", en GUYER, P. (ed.): The Cambridge Companion to Kant, Cambridge, Cambridge University Press, 1992. 
ZOCHER, Rudolf: “Zu Kants transzendentaler Deduktion der Ideen der reinen Vernunft”, Zeitschrift für philosophische Forschung 12 (1958) 43-58.

ZOCHER, Rudolf: "Der Doppelsinn der kantischen Ideenlehre. Eine Problemstellung”, Zeitschrift für philosophische Forschung 20 (1966) 222-226. 